Sociologie et sociétés

SOCIOLOGIE

ET SOCIÉTÉS

\title{
Une américanité cosmopolite
}

Pour la suite d'un débat

\section{Cosmopolitan Americanity.}

An Ongoing Debate

\section{Jean-François Côté}

Volume 38, numéro 2, automne 2006

Michel Foucault : sociologue?

URI : https://id.erudit.org/iderudit/016382ar

DOI : https://doi.org/10.7202/016382ar

Aller au sommaire du numéro

\section{Éditeur(s)}

Les Presses de l'Université de Montréal

\section{ISSN}

0038-030X (imprimé)

1492-1375 (numérique)

Découvrir la revue

Citer ce document

Côté, J.-F. (2006). Une américanité cosmopolite : pour la suite d'un débat.

Sociologie et sociétés, 38(2), 243-251. https://doi.org/10.7202/016382ar 


\title{
Une américanité cosmopolite
}

Pour la suite d'un débat

\author{
JEAN-FRANÇOIS CÔTÉ \\ Université du Québec à Montréal \\ Département de sociologie \\ C.P. 8888 , Succursale Centre-ville \\ Montréal, Québec, Canada $-\mathrm{H}_{3} \mathrm{C}_{3} \mathrm{P} 8$ \\ Courriel: cote.jean-francois@uqam.ca
}

T a question de L'américanité continue d'habiter l'horizon de la réflexion au Québec, et le débat de fond soulevé par cette question, qui s'est poursuivi dans les pages de cette revue (Miguelez, 2004; Thériault, 2004), m’incite à répondre à l'invitation au moins implicite qui m'est faite de m'y engager ${ }^{1}$. Pour ce faire, je tenterai de déceler, surtout à travers deux dimensions (idéologique et sociologique), des points de désaccord profonds avec l'analyse proposée par Thériault (2002), qui a servi à lancer le débat autour de cette question sur des bases polémiques.

À cet égard, je confesse d'abord et avant tout un malaise sérieux ressenti à la lecture du livre de Thériault (malaise ressenti initialement, mais toujours présent), qui me semble préfigurer bon nombre de complications superflues dans le débat; ce malaise tient à une formulation qui apparaît fréquemment dans l'ouvrage, et qui conduit à mes yeux à une mauvaise appréhension fondamentale de cette question de l'américanité. On trouve en effet assez souvent, au fil des pages de Critique de l'américanité, une structure grammaticale qui conduit l'«objet» de l'interrogation à se transformer en «sujet»,

1. Dans son article qui réplique à Miguelez, Thériault fait référence à un article que j'avais moi-même écrit sur la question (Thériault, 2004, p. 266; Côté, 2001a), comme il l'avait fait auparavant dans son ouvrage (Thériault, 2002, p. 85, 356-357). Je reviendrai plus loin sur ceci. 
comme par exemple lorsqu'on lit: «L'américanité s'affirme au départ comme l'idéologie anti-canadienne-française par excellence», ou encore: «En extirpant de l'Amérique ce qui est américain, l'américanité dresse un portrait d'une Amérique sans âme, idéaltype de la modernité radicale avant d'être celui d'une société réelle» (2002, p. 24, 58; je souligne $)^{2}$. Ce qui est embêtant dans de telles formulations, c'est qu'elles empêchent de voir que l'on parle véritablement de l' «objet» de la réflexion de la part d'un «sujet» quelconque de cette réflexion, comme si l'on présumait en fait que tous les sujets qui prennent l' «américanité» comme objet de réflexion le faisaient exactement de la même manière, sans possibilité de repérer des différences de position à son égard, et sans possibilité que l'on situe plus exactement l'«objet» que ces sujets tentent eux-mêmes de cerner dans leur propre réflexion - et sans que l'on puisse tout autant entrevoir finalement la forme de relation entre le ou les sujets de la réflexion et leur ou leurs objets comme étant au centre de l'examen proposé. Dans la (con)fusion entre le sujet, l'objet, et la forme de leur relation dans la réflexion, il n'est donc pas étonnant que l'on rencontre le point de départ de l'analyse dans cette formule lapidaire:«L'américanité est un concept-poubelle. Poubelle dans le sens d'un ramassis hétéroclite d'énoncés dont on réussit difficilement à trouver la forme» (Thériault, 2002, p. 23). En effet. En l'absence de distinctions élémentaires sur la structure des énonciations qui empêchent de repérer les places respectives du sujet, de l'objet, ainsi que la forme révélant le contenu de leur relation, nous sommes dans la plus totale confusion ${ }^{3}$. Cela peut s'expliquer, à mon sens, uniquement du point de vue où le parti pris de Thériault est illustré par le fait de considérer comme une idéologie, sinon comme une mode ou un «large consensus», cette question de l'américanité, sans faire l'effort préalable de lui accorder le statut d'objet de réflexion légitime. Or, je rappelle que l' «américanité», comme objet de réflexion, pose avant tout la question de l' «identité américaine», c'est-à-dire, selon une formulation que je serai appelé pour ma part à détailler plus bas, une interrogation sur l' «identité des Amériques» — que Thériault réduit par ailleurs aux États-Unis, tout comme certaines des réflexions produites dans ce contexte qu'il examine. Qu'une telle interrogation puisse être réfléchie au Québec, comme partout ailleurs dans les Amériques (et même ailleurs dans le monde), me semble justement non seulement légitime, mais même nécessaire, surtout dans le contexte contemporain.

\section{DE L'IDÉOLOGIE...}

Thériault présente l' «américanité», dans sa dimension uniquement idéologique (mais cela n'est pas distingué d'autres possibilités, et plutôt assimilé rapidement à une «absence d'intentionnalité» — paradoxe sur lequel nous reviendrons sous peu), comme un renoncement, ou comme un échec, alors que, dans ce contexte à tout le moins, c'est

2. Sans en faire le relevé exhaustif, on trouve d'autres formulations semblables aux pages 13, 27, 29, $39,40,63,86,101,122,152,159,341$, etc.

3. Confusion totale qu'évite par ailleurs Thériault, en s'appliquant justement la plupart du temps à l'examen de positions déterminées; le problème est cependant qu'il rapporte toutes celles-ci à la thématique de l'américanité traitée alors, étrangement, de manière unilatérale. 
à mon sens précisément l'inverse: la majorité des discours et expressions qui prennent l'américanité en tant que thématique idéologique apparaissent, faut-il le rappeler, après le projet «canadien-français», et même après le projet "québécois» — mais dans ce dernier cas le après doit être relativisé, puisque ce n'est pas d'une séquence, mais pratiquement d'une simultanéité dont il doit être question, étant donné que la thématique de l'américanité apparaît bien dans la foulée du développement de l'identité québécoise, moins comme son substitut en fait (ce que les thuriféraires de la québécitude voudraient qu'il soit) que comme complément, ou son prolongement au sein d'une actualité politique qui ne requiert certaines idées que lorsqu'elle est prête à les accueillir $^{4}$. Prolongement continental, dans un sens, mais qu'on pourrait tout aussi bien voir comme appréhension d'une «extériorité» politique par rapport à l' «intériorité» de l'identité nationale québécoise. Car c'est également de cela qu'il s'agit; si, en l'espace d'une vingtaine d'années à peine, l'intégration continentale a eu un effet très significatif sur l'économie québécoise, et sur l'ensemble de la société, n'est-il pas simplement au moins normal que cette question se réfléchisse dans la conscience politique? Il ne s'agit pas ici d'applaudir à la vue de ces réalités — intention que me prête, semble-t-il, Thériault (2004, p. 266;2002, p. 85) —, mais bien de tenter de les envisager pour ce qu'elles sont, dans un premier temps, puis de s'engager si possible dans une réflexion plus approfondie sur le sujet, au risque, si on ne le fait pas, de laisser précisément toute la question dans un impensé de la réflexion, politique d'abord (sur le plan idéologique) et éventuellement sociologique. Dans ce sens, la thématique idéologique de l'américanité, en tant qu'elle prend part aux discussions concernant les divers projets d'intégration continentale (de manière positive ou critique) ne peut pas être dénuée $\mathrm{d}$ ' «intentionnalité » — à moins de voir paradoxalement dans toute idéologie une... «absence d'intentionnalité » — et n'est pas davantage un "échec», un projet «mort-né», et encore moins un renoncement, comme la présente Thériault, c'est plutôt justement et au contraire la poursuite d'une certaine tradition politique, soit celle qui conduit la société d'ici sur des voies nouvelles si l'on veut, mais en autant, bien entendu, que l'on veuille également voir dans le mot «tradition» autre chose qu'une répétition stérile d'un passé devenu obsolète - c'est-à-dire donc comme une traduction active de ce passé dans quelque chose qui est susceptible de lui fournir une forme d'expression renouvelée. La tradition, c'est avant tout cela, c'est-à-dire encore, comme le souligne à juste titre Hans-Georg Gadamer (1996), une expérience susceptible de s'ouvrir à son renouvellement - et non pas une expérience fermée sur elle-même et enfermée définitivement dans ses anciennes limites, figées dans un modèle qu'on prétend pouvoir reproduire fidèlement et éternellement (cela, je le fais d'ailleurs remarquer en passant, c'est le propre du mythe). Nous touchons ici la dimension proprement sociologique de

4. Et je dis cela pour bien marquer le fait que la question de l'américanité avait aussi été posée dans différentes expressions - sociologique (Guy Rocher), géographique (Jean Morrisset), artistique (Robert Charlebois, Jacques Languirand, Jacques Godbout), etc. — avant qu'elle ne circule à plus large échelle, comme thématique idéologique, dans le débat politique au Québec. 
l'analyse, mais avant d'avant d'aborder cette dimension, je relève un aspect peut-être plus cocasse des choses. Ce très court excursus sera d'ailleurs ici, je l'espère, éclairant.

Bien sûr, Maria Chapdelaine appartient d'une certaine manière au passé (littéraire) «canadien-français» — mâtinée rapidement toutefois d'une certaine vision européenne, le Français d'origine bretonne Louis Hémon ayant comme on le sait profité de son court passage au Québec (moins de deux années), situé entre ses pérégrinations londoniennes, qui dureront huit ans, et l'appel de son aventure écourtée vers l'ouest du continent nord-américain (il mourra accidentellement à Chapleau, en Ontario, en 1913), pour travailler ce feuilleton, publié finalement sous forme de roman de manière posthume. Lorsque Louis Hémon travaille à la publication des épisodes de ce qui deviendra son Maria Chapdelaine, c'est au retour en fait d'un hiver passé dans la région du Lac-Saint-Jean en 1912, ce qui le ramène à Montréal pour mettre en forme son manuscrit, lui qui n'a jamais interrompu sa vie de nomade; relire aujourd'hui ce roman en le situant dans ces entrelacs biographiques, littéraires, contextuels et événementiels serait un pari analytique intéressant, mais ne donnant certainement pas lieu à l'ancrage symbolique absolu dont Thériault veut le lester dans son ouvrage, en en faisant pratiquement le roman éponyme des Canadiens-français. Or, et ceci étant dit, on ne peut tout de même pas être aveugle au point de consentir à ne pas voir que nous sommes aujourd'hui dans un horizon de sens complètement différent ${ }^{5}$. Ou le peuton? Avec Thériault, il semble en tout cas que oui ${ }^{6}$. Car c'est précisément à l'enseigne de la Maria Chapdelaine de Louis Hémon qu'il place son plaidoyer en faveur d'une critique intrinsèque et pour ainsi dire «réellement vécue» de l'américanité — l'ironie du sort voulant que ce roman soit devenu, aussi, un best-seller dans sa traduction anglaise dans les années $1920 \ldots$ notamment aux États-Unis ${ }^{7}$.

5. On devrait d'ailleurs souligner que cet horizon, rapporté au traditionalisme canadien-français, était déjà, au moment même où Hémon en donnait une représentation littéraire, marginalisé par le développement de la société québécoise (c'est-à-dire circonscrite au Québec, mais non seulement «canadiennefrançaise») de l'époque, largement ouverte sur l'industrialisation, et que c'est justement du côté de cette «excentricité» d'un récit situé sur la rivière Péribonka qu'il pouvait trouver ancrage. Hémon eût-il choisi Québec ou Montréal comme situation de son récit, la représentation de l'«horizon canadien-français» (majoritaire cette fois, et non plus seulement excentrique ou marginalisé par rapport à la situation de l'époque) aurait sans doute été passablement différente. D'ailleurs, pour saisir d'une manière sociologique la signification de ce roman, ne faudrait-il pas au moins l'inscrire parmi les autres représentations romanesques du temps, d'abord, puis s'interroger ensuite au moins sur ses caractéristiques esthétiques formelles?

6. La lecture que fait Thériault (2002, p. 51-52) du roman de Hémon, qu'il situe dans la lignée des «romans de la terre» allant de Philippe Aubert de Gaspé à Félix-Antoine Savard, lui fait se reporter aux remarques de Tocqueville qui notait, dans les années 1830, le caractère «traditionaliste» et presque féodal du Bas-Canada, opposé radicalement dans ce sens aux États-Unis; mais le roman de Hémon étant publié au début du $\mathrm{xx}^{\mathrm{e}}$ siècle, doit-on comprendre que rien n’a alors changé - et surtout que la représentation romanesque tient au surplus à un «réalisme» sur lequel on peut appuyer une vision «objective» de la société canadienne-française du temps?

7. À cet égard, et du point de vue d'une sociologie de la réception, il faudrait aussi s'interroger sur les raisons qui font qu'un tel roman, à saveur «traditionaliste», trouve un si grand écho - le roman a été traduit depuis en une vingtaine de langues — du côté de bon nombre de sociétés alors en pleine industrialisation et en phase d'intense urbanisation, sans que ce soit la spécificité locale canadienne-française qui trouve là sa résonance particulière (et ceci en dehors du fait évidemment qu'il joue alors strictement son rôle de 


\section{... À LA SOCIOLOGIE}

La manière par laquelle s'articule la thématique de l'américanité à cette interrogation sur l'histoire de la tradition canadienne-française, je la vois pour ma part justement et entre autres dans le dialogue implicite et explicite qui s'est noué au fil de l'histoire avec les différents acteurs présents sur le continent, parce que c'est précisément là un élément déterminant de son identité profonde, qu'on l'envisage soit dans son rapport aux métropoles européennes française et anglaise, soit dans son rapport refoulé aux autochtones, soit dans son rapport au pacte politique canadien, soit dans ses rapports croissants avec les autres sociétés du continent (Côté, 2001b). Reléguer la signification de cette appartenance continentale à l' «évidence» ou à la «banalité» (Thériault, 2002, p. 12), c'est, au mieux, ignorer la portée de la dimension géopolitique d'existence d'une société - en la situant ainsi spatialement dans les limbes - et, au pire, renoncer volontairement à s'ouvrir à un questionnement sur ce plan. Et ce questionnement doit alors prendre à son compte des considérations disciplinaires et épistémologiques.

La deuxième partie de l'ouvrage de Thériault, qui est de loin à mes yeux la plus forte, s'appuie ainsi essentiellement sur la distinction épistémologique wébérienne entre « expliquer» et «comprendre» — une distinction que la théorie herméneutique contemporaine a largement dépassée en proposant plutôt, justement, une théorie de l'interprétation, qui a l'avantage de reprendre à son compte à la fois les principes de la compréhension et ceux de l'explication, en les subsumant, pourrait-on dire, sous le registre non pas d'une alternative, mais bien plutôt d'une complémentarité obligée, dans laquelle la positivité de la compréhension — soit la prise en compte de tous les éléments devant rendre compte de ce qui est en jeu - s'accomplit dans la critique de l'explication - soit la mise en évidence des relations d'implication réciproque entre ces éléments (une mise en évidence qui met en scène la «causalité», mais aussi et surtout, dans le contexte des sciences humaines, la "plausibilité » de ces relations). L'interprétation, quant à elle, et dans la mesure où elle fait siennes explication et compréhension dans l'orbe du cercle herméneutique, se suffit de sa propre historicité, c'està-dire du fait qu'elle n'apparaît pas comme ultime et indépassable, mais plutôt comme satisfaisant aux exigences (rigoureuses par ailleurs) de son propre temps, c'est-à-dire donc d'une compréhension des éléments qu'elle aborde et d'une explication de leurs rapports logiques d'emboîtement réciproque. C'est de ce point de vue que l'interprétation, dans la théorie herméneutique, participe réflexivement au développement de la tradition: par une actualisation dans laquelle elle se met en jeu au sein d'un processus susceptible de faire apparaître les bornes ou les limites de la pré-compréhension (du "préjugé», comme dit Gadamer) dont elle avait hérité, afin de reconduire la signification sur laquelle elle se penche au-delà de son contexte d'émergence original et initial. Le débat sur l'historiographie québécoise récente dans lequel s'inscrit la critique de Thériault est, à cet égard, réellement intéressant — mais peut-être pas cependant pour

roman, et donc de projection imaginaire par rapport à la réalité, ce qui n'exclut évidemment pas une teneur «nostalgique» par exemple). 
les motifs invoqués dans sa «relecture», qui vise essentiellement ici à montrer que l'on aurait privilégié indûment l' "explication» au détriment de la «compréhension», c'està-dire les grands processus rejoignant la société québécoise qui négligent la particularité de sa situation. Ici, et la question avait été soulevée par l'ouvrage de Ronald Rudin (1998) auquel se réfère principalement Thériault, la relecture «révisionniste» de l'historiographie qui ferait la part trop belle aux «processus» par rapport au «vécu» de la société paraît négliger l'«intentionnalité» d'une société canadienne-française poursuivant, au-delà des développements (bien sûr «triviaux»...) du capitalisme contemporain, les espoirs de sa propre «subjectivité» historique — dans une opposition ontologique bien trop claire ici entre «matériel» et «spirituel» qui nous ramène à une mentalité que l'on connaît bien. Le débat, présenté sous cet angle, demanderait ainsi beaucoup plus qu'une simple contextualisation des rivalités entre l' «École de Québec» et l'«École de Montréal» sur lequel il a pris forme dans les années 1940, 1950 et 1960, et depuis, puisque, en réalité, il fait ressortir les tréfonds de l'entreprise historiographique québécoise de la période précédente, disons grosso modo depuis François-Xavier Garneau jusqu'à Lionel Groulx. C'est là en effet, comme on le sait, que s'est logée, dans toute son ampleur et dans toute son ambiguïté, la question d'une «histoire nationale» canadienne-française appelée éventuellement à devenir québécoise, et que serait apparue l' «intentionnalité» si chère à Thériault. Peu importe à cet égard pour lui, semblet-il, que cet arrière-plan ait été nourri, voire soutenu, par une téléologie historique d'inspiration proprement et essentiellement théologique (et ici Thériault suit Fernand Dumont de près, particulièrement dans les limites et le relatif aveuglement de ce dernier par rapport au développement et à l'évolution de la société «canadienne-française» — voir Côté, 2001c), car l'idée ici est de montrer que c'est justement cette histoire-là qui correspondait... à l'orientation idéologique de l'époque, arc-boutée sur des positions (évidemment compréhensibles par ailleurs à ce moment) visant la pleine reconnaissance du Canada français par le Canada anglais. C'est donc contre la révision plus récente de cette historiographie que s'élève Thériault (davantage que Rudin au fait, qui ne faisait, lui, que relever cette situation, pour la questionner, et non pas nécessairement pour la condamner...). Ici, la lecture de ces débats par Thériault vise principalement non pas à permettre une évaluation sociologique de ces questions, mais à entériner une des positions en cause — soit celle qui permettrait que l'on «comprenne» l'histoire du Canada français (sans l'«expliquer»... ?) à la lumière de son indépassable singularité, une histoire située même à la limite à l'écart en quelque sorte des grands mouvements de l'histoire contemporaine, quitte à s'abstraire de celle-ci afin de permettre la lecture d'une spécificité qui s'est construite justement dans un lieu épargné par à peu près tous les événements historiques touchant le monde à l'extérieur d'elle de nouveau, dans les limbes, non pas de l'espace cette fois-ci mais bien du temps, de l'histoire donc, cette position si confortable dans laquelle on aime parfois situer la société québécoise pour éviter de s'interroger vraiment à son sujet ou, ce qui revient à peu près au même, pour l'enfermer définitivement dans la particularité («spirituelle» ?...) de son cas. Or, un rapide coup d'œil à l'extérieur des étroites limites fixées 
par Thériault montre que c'est partout dans les Amériques que s'ébauchaient alors les historiographies nationales - ce qui n'est pas une très grande surprise, du fait que c'est justement dans cette période que s'affirmaient les nouvelles réalités nationales sur tout le continent. En faisant remarquer à Thériault ce dernier point de la constitution pour ainsi dire a posteriori des représentations nationales dans le Nouveau Monde par rapport à l'apparition de leurs structures politiques étatiques, Roberto Miguelez (2004, p. 255-257) montre bien, à mon sens, que par rapport à l' «intentionnalité» canadiennefrançaise, d'autres «intentionnalités» tout aussi légitimes ( $y$ compris celle des ÉtatsUnis) prenaient forme au sein d'une communauté de préoccupations visant la définition de spécificités nationales locales au sein des Amériques.

Davantage, même, on devrait souligner que l'«intentionnalité» qui apparaît dans le contexte de la formation des sociétés nationales des Amériques ne peut le faire qu'après que se soit estompée l' "intentionnalité» européenne première à la source de la colonisation d'ensemble des populations autochtones (ou même, les différentes «intentionnalités» qui avaient cours au sein de l'Europe, et qui partageaient par exemple les visées de l'Espagne, du Portugal, de l'Angleterre, de la France, etc., en projets de colonisation des Amériques possédant chacun des caractéristiques propres, et produisant finalement des modes d'occupation du territoire et des modes de structuration de la société somme toute assez différents) ${ }^{8}$. Et que se soit par le fait même aussi estompée l'«intentionnalité» (ou les «intentionnalités») dont étaient porteuses les sociétés autochtones — dont on devrait plutôt dire qu'elles se la sont fait estropier ${ }^{9}$.

Je dirais toutefois à cet égard que la terminologie employée par Thériault ne me semble pas convaincante. Car pour réaliser un examen vraiment sérieux des choses, il faudrait revenir en fait ici aux bases philosophiques qui ont permis à Jürgen Habermas d'emprunter cette terminologie husserlienne en lui faisant servir de tout autres destinations que celles qu'elles servaient chez Husserl lui-même — dans l'opposition «système» et «monde vécu», destinations désormais tout habermassiennes, que je considère pour ma part comme étant simplement erronées (Côté, 1998). À mes yeux, ici aussi reposent une certaine confusion et certains contresens qu'il vaudrait la peine d'examiner plus en détail, avant de pouvoir les transposer à nouveau dans le contexte d'une analyse de l'historiographie québécoise, ou encore de l'analytique de la question de l'américanité.

8. Bien sûr, apparaît ici toute la question de l'institutionnalisation de la modernité, qui se fait de manière parallèle en Europe et dans les Amériques, mais qui appellerait, précisément pour cette raison, une analyse comparative très attentive des processus qu'elle génère dans ces contextes respectifs. Ici, et en suivant de nouveau les remarques de Miguelez (2002) sur cette question, je crois qu'une plus grande «communauté» de préoccupations se fait jour à travers les Amériques, même en fonction de la particularité des cas, dans le rapport désormais dual qui s'instaure avec l'Europe.

9. Si l'on accepte de s'ouvrir sur cette possibilité d' «anthropologiser» la notion d' «intentionnalité» de Husserl, il faut entrevoir son application en dehors de la tradition occidentale à laquelle lui-même la rattachait essentiellement, pour la considérer dans ses liens aux grandes formes de représentation que sont les mythes, les religions, et la science — formes expressives dont l'égalité de contenu (spirituel, en dépit de leurs différences de formes, précisément) a été reconnue par la sociologie de Durkheim et de Weber, notamment, après qu'elle l'eut été dans différents registres par Vico, Hegel et Humbolt, entre autres. 


\section{UNE AMÉRICANITÉ COSMOPOLITE}

À l'encontre de ce que croit Thériault, réfléchir à la question de l'américanité peut impliquer, et c'est ce que je tente en tout cas pour ma part de faire, de chercher une position analytique permettant de contredire l'idée que l' «américanité québécoise» n'est pas le projet d'inventorier les multiples manières nationales d'être en Amérique, mais bien celui de concevoir, au-delà des différences, une «appartenance continentale» (2002, p. 31). Je crois de mon côté que les deux choses sont simultanément possibles, qu'elles ne sont aucunement contradictoires, et même au contraire très compatibles. Je crois en effet que l'analyse comparative de certaines formes d'expressions issues de contextes nationaux (telle la littérature, mais pas seulement elle, bien entendu) peut nous aider à saisir des enjeux de définitions identitaires à la fois nationaux et continentaux (Côté et Tremblay, 2005). Je crois même qu'on a avantage à réexaminer de cette façon toute l'histoire des Amériques, depuis ses origines jusqu'à ses développements récents. On déplace ainsi en quelque sorte l'horizon d'interrogation des significations en jeu, pour appréhender par exemple la définition de formes de cosmopolitisme qui ont et auraient eu prise bien en deçà (et bien au-delà) de la manière par laquelle les transformations en cours dans les différents processus d'intégration continentale se posent actuellement, en fonction des très étroites prérogatives politicoéconomiques de l'idéologie néolibérale. Ces formes de cosmopolitisme, liées à l'identité continentale des Amériques en fonction d'abord des «rencontres» initiales entre populations européennes et autochtones (puis africaines), ensuite de leurs ancrages nationaux respectifs (nourris également des diverses immigrations subséquentes), peuvent nous informer sur les possibilités plus larges de (re)définition d'une communauté politique américaine qui dépasserait les définitions qui en sont données actuellement. Ces définitions doivent toujours, bien entendu, compter avec leurs possibilités et leurs limites respectives, et c'est la raison pour laquelle elles posent la question de l'identité américaine à travers tout le continent. Et elles doivent tout aussi bien composer avec une réalité imposante, qui a fait par exemple des États-Unis un acteur majeur dans ce contexte, par son implication directe dans l'établissement de relations (politiques, économiques, militaires, culturelles, etc.). Mais si la forme impériale qui s'est développée aux États-Unis, surtout entre le premier quart du $\mathrm{XIX}^{\mathrm{e}}$ siècle et le dernier quart $\mathrm{du} \mathrm{Xx}^{\mathrm{e}}$, continue toujours d'être un problème sociologique à réfléchir, elle doit elle-même aussi s'articuler autour d'une définition de l'identité nationale étatsunienne en profonde transformation. En effet, ce n'est pas en ce moment que dans le contexte québécois que se pose la question de l'identité des Amériques, avec ses répercussions sur le plan national, bien que le débat sur l'américanité qui a cours ici présente indubitablement des indications et des possibilités qui sont intrinsèquement liées aux conditions que nous rencontrons, en tant qu'intellectuels et en tant que citoyens d'ici. Il est difficile de prévoir l'évolution prochaine d'une reconnaissance de l'identité symbolique d'ensemble des Amériques, ainsi que l'ensemble de ses répercussions, mais cette évolution ne pourra en aucun cas se faire sans nous, que nous en soyons ou non conscients, et que nous acceptions ou non d'en 
discuter comme d'une chose de l'esprit qui nous appartient de façon pleinement légitime.

\section{BIBLIOGRAPHIE}

Côté, J.-F et E. Tremblay (dir.) (2005), Le nouveau récit des frontières dans les Amériques, Québec, Presses de l'Université Laval.

Côté, J.-F. (2001a), «L'identification américaine au Québec: de processus en résultats», dans Donald Cuccioletta (dir.), L'américanité et les Amériques, Québec, Éditions de l'IQRC, Presses de l'Université Laval, p. 6-27.

Côté, J.-F. (2001b), «Le renouveau du grand récit des Amériques: polyphonie de l'identité culturelle dans le contexte de la continentalisation », dans D. Cuccioletta, J.-F. Côté et F. Lesemann (dir.), Le grand récit des Amériques, Éditions de l'IQRC, Presses de l’Université Laval, p. 9-37.

Côté, J.-F. (2001c), Compte rendu de Fernand Dumont, Chantiers. Essais sur la pratique des sciences de l'homme, Montréal, Hurtubise, HMH, 1973, 253 p., Recherches sociographiques, vol. XLII, nº 2, p. 372377.

Côté, J.-F. (1998), «L’opposition entre “système” et “monde vécu” dans la sociologie de Jürgen Habermas et la médiation idéologique de la communication dans la société contemporaine», Carrefour, 20, 1, p. 37-55.

Gadamer, H.-G. (1996), Vérité et méthode, trad. Jean Grondin et Gilbert Merlio, Paris, Seuil.

Miguelez, R. (2004), "À propos de la Critique de l'américanité de Joseph Yvon Thériault», Sociologie et sociétés, vol. XXXVI, $\mathrm{n}^{\circ}$ 2, p. 247-259

Rudin, R. (1998), Faire de l'histoire au Québec, trad. Pierre R. Desrosiers, Québec, Septentrion.

Thériault, J. Y. (2004), "Réponse à la critique de la Critique de l'américanité», Sociologie et sociétés, vol. XXXVI, n ${ }^{\circ}$ 2, p. 261-267.

Thériault, J. Y. (2002), Critique de l'américanité. Mémoire et démocratie au Québec, Montréal, Éditions Québec Amérique. 